oder Traubenzuckers bei weitem vorzuziehen. Aus der vom Platinsulfid getrennten Flüssigkeit ist nun das $\mathrm{Li}$ thion zu gewinnen. Dies geschieht, indem man dieselbe concentrirt und das Lithion mit kohlensaurem $\mathrm{N}$ atron als kohlensaures Salz fällt. Hat man hierbei vorber das $\mathrm{NaCl}$ und $\mathrm{KCl}$ durch Herauskrystallisiren grösstentheils entfernt und das Ammoniak durch Zusatz von Aetznatron vertrieben, so kann man die Mutterlauge, aus der das Lithion gefällt wurde und die immer noch etwas Lithion neben überschüssiger $\mathrm{NaO}, \mathrm{CO}^{2}$ enthält, bei der nächsten Operation wieder verwenden. Hinsichtlich der Bearbeitung des Glimmers von Zinnwald gilt was vom Lepidolith angeführt wurde, da beide Mineralien im Ganzen genommen, dasselbe Verhalten zeigen. Der Glimmer schmilzt jedoch leichter, schäumt dabei weniger auf als der Lepidolith und giebt eine dunkelgrïne glasartige Masse, deren Dichte 2,27 beträgt. Da derselbe weit mehr Eisen enthält als der Lepidolith, so ist es vortheilhafter, die Oxydation desselben mit unterschlorigsaurem Natron als mit $\mathrm{NO}^{5}$ zu bewirken. Die weitere Behandlung bleibt dieselbe.

Nach Schrötter's Analysen enthält der Lepidolith 3,19 Proc. Lithion (= 1,476 Proc. Lithium entsprechend 7,8 Proc. $\left.\mathrm{LiO}, \mathrm{CO}^{2}\right), 0,536$ Proc. cäsiumhaltiges Rubidiumoxyd und 0,006 Proc. Thallium. 100 Th. quarzfreier Lepidolith enthalten nach ihm 51,746 Th. Kieselerde und erleiden beim Schmelzen 1,513 Proc. Glühverlust. Im quarzfreien Glimmer von Zinnwald fand Schrötter 49,782 Proc. Kieselerde, 0,75 Proc. cäsiumhaltiges Rubidium, 0,814 Proc. Lithium und 0,0065 Procent Thallium. Das hieraus gewonnene Rubidium ist reicher an Cäsium als das aus Lepidolith gewonnene. (Wiener Sitzungsber. d. k. Akad. d. Wissensch. Math.-naturv. Classe. L. Bd. II. Heft. II. Abth. S. 268-284.) H. Ludwig.

\title{
Ueber die Trennung von Rubidium und Cäsinm in Form der Alaune
}

hat Joseph Redtenbacher Versuche veröffentlicht.

Die bisherige Trennungsmethode von $\mathrm{K}, \mathrm{Rb}$ und $\mathrm{Cs}$ beruht auf der verschiedenen Löslicbkeit der Platindoppelsalze. Diese von Bunsen, dem Entdecker dieser Metalle, zuerst gefundene Methode bietet die Schwierigkeit der Schwerlöslichkeit der genannten Platinsalze, 
so dass, wenn man grössere Mengen von Material beàrbeitet, man grosse Mengen von Flüssigkeit zu bewältigen hat und dass, selbst wenn man fractionirt arbeitet, die Anwendung von Platin den Vorgang kostspielig macht und die fabrikmässige Darstellung von vornherein bindert.

Die Trennung als weinsaure Salze setzt die vorherige Anwendung der Platinsalze voraus.

Redtenbacher versuchte, ob nicht die verschiedenen Alaune von $\mathrm{K}, \mathrm{Rb}$ und $\mathrm{Cs}$ durch fractionirte Krystallisation sich trennen liessen und diese Versuche gelangen.

In der chemischen Fabrik des Herrn Dr: Würth stellt Dr. Schorm Lithionpräparate aus dem mährischen Lepidolith von Roczna in grossem Massstaive dar. Von ihnen erhielt Redtenbacher das Material zu seinen Versuchen. Bei Bearbeitung der Rückstände, aus welchen das $\mathrm{LiCl}$ ausgezogen war, um daraus mit Platinsalz das Cs und $R b$ zu gewinnen, fiel die Ausbeute, den bisherigen Erfahrungen entgegen, so geringe aus, dass auf einen Verlust dieser Metalle in den Operationen der Fabrik geschlossen werden musste, da auf einen ungeheueren Ueberschuss von Kalium ganz unbeträchtliche Mengen von Rubidium und nur Spuren von Cäsium sich vorfanden. Der Lepidolith wird in der genannten Fabrik mit $\mathrm{S}$ ch wefelsäure aufgeschlossen, die geglühte Masse mit Wasser ausgezogen, die wässerige Lösung zur Krystallisation eingedampft, um die meiste Thonerde als Alaun zu entfernen. Wenn man die Schwerlöslichkeit der Alaune im Verhältnisse der Zunabme des Aequivalents ebenfalls zunimmt, so mussten in den ersten Alaunkrystallisationen obiger Fabrik sich vorzüglich Rubidium und Cäsium fin. den. Die ersten Alaunproben, welche Redtenbacher aus der Fabrik erhielt, gaben schon obne rorheriges Umkyystallisiren das deutlichste Spectrum von Rubidium und Cäsium.

Es wurden hierauf $37 \mathrm{Pfd}$. Alaun in Arbeit genommen und daraus durch eine Reihe von Krystallisationen aus Wasser $1 / 4$ Pfd. reiner Cäsiumalaun, 1/2 Pfd. reiner Rubidiumalaun und 2 Pfd. eines vollkommen kalifreien Gemenges von Rubidium- und Cäsiumalaun erhalten, welches erst später aufgearbeitet werden soll. In der Mutterlauge fand sich als das leichtest lösliche Salz auch der Thalliumalaun mit starkem Eisenoxydgehalte.

Bei dieser Veranlassung wurden einige Eigenschaften des Rubidium- und Cäsiumalauns näher untersucht. Schon Bunsen beobachtete die Krystallgestalt derselben als 
tesseral. Dr. Tschermak untersuchte ausgesuchte Krystalle derselben, welche ihm Redtenbacher hierzu übergeben hatte.

Die Krystalle des Rubidiumalaunes sind reguläre Octaëder, deren Kanten und Ecken durch die Flächen des Rhombendodekaëders und Hexaëders schwach abgestumpft erscheinen; nur selten sieht man die Flächen des Pentagonaldodekaëders.

Der Cäsiumalaun zeigt ebenfalls tesserale Krystalle, die oft sehr flächenreich sind, dabei aber ebenfalls immer die Flächen des Octaëders aufweisen. Eine häufige Combination ist die des Octaëders mit dem Pentago. naldodekaëder, in welcher sich die beiden Formen oft das Gleichgewicht halten, so dass die bekannte mit 20 Dreiecken begrenzte Gestalt ergiebt. Sonst sieht man das Octaëder mit den Flächen des Hexaëders, Pentagonaldodekaëders und mit dem Ikosetetraëders.

Eigenthümlich ist für den Cäsiumalaun das beständige Auftreten der Flächen des Pentagonaldodekaëders, die bei dem Kalialaun nur ausnahmsweise vorkommen, wenn derselbe aus einer salzsauren Lösung krystallisirt.

Das specifische Gewicht von Cäsiumalaun wurde $=$ 2,003 gefunden, das des Rubidiumalauns $=1,874$, während $\mathrm{K}$ op $\mathrm{p}$ das des Kalialauns $=1,724$ fand. So wie das Aequivalent des $\mathrm{Rb}$ das mittlere von dem des $\mathrm{K}$ und Cs ist, so ist dasselbe Verhältniss in der Dichte ihrer Alaune, denn $\frac{1,724+2,003}{2}=1,863$.

Beide Alaune sind in kochendheissem Wasser sehr leicht löslich, dafür aber in kaltem Wasser viel schwerer löslich als Kalialaun. 100 Th. Wasser lösen bei $16^{\circ} \mathrm{C} .: 2,271 \mathrm{Th}$. Rubidiumalaun, oder $1 \mathrm{Th}$. Rubidiumalaun löst sich bei $16^{\circ} \mathrm{C}$. in $44 \mathrm{Th}$. Wasser und die Lösung hat ein spec. Gew. $=1,0125$.

$100 \mathrm{Th}$. Wasser bei $17^{0} \mathrm{C}$. lösen $0,6188 \mathrm{Th}$. Cäsiumalaun, oder $1 \mathrm{Th}$. Cäsiumalaun löst sich bei $170 \mathrm{C}$. in $160 \mathrm{Th}$. Wasser und die gesättigte Lösung hat bei $17^{\circ} \mathrm{C}$. ein spec. Gew. $=1,0036$. Poggiale fand für Kalialaun folgende Zahlen:

$100 \mathrm{Th}$. Wasser bei $17^{0} \mathrm{C}$. lösen $13,5 \mathrm{Th}$. Kali . alaun, oder $1 \mathrm{Th}$. Kalialaun bei $17^{\circ} \mathrm{C}$. löst sich in 7,4 Theile Wasser.

Die Löslichkeit dieser 3 Alaune bei $17^{0} \mathrm{C}$. verhält sich zu einander wie

$$
13,5(\mathrm{~K}): 2,27(\mathrm{Rb}): 0,6188(\mathrm{Cs})
$$

Arch. d. Pharm. CLXXVII. Bds. 1. u. 2. Hft. 
oder in ganzen Zahlen ausgedrückt wie $22(\mathrm{~K}): 4(\mathrm{Rb}): 1(\mathrm{Cs})$

während die Löslichkeit der Platinsalze dieser Metalle sich nach $B$ unsen verhält

$$
\begin{aligned}
& \mathrm{K}: \mathrm{Rb}: \mathrm{Cs} \\
& \text { bei } 17^{\circ} \mathrm{C} .15: 2: 1 \\
& \text { n } 100^{\circ} \text { C. } 14: 1,7: 1 \text {. }
\end{aligned}
$$

Die Alaune bieten also den Vortheil, dass sie in heissem Wasser sehr leicht löslich, in kaltem Wasser aber ziemlich schwerlöslich und dass ihre Löslichkeitsdifferenzen grösser sind, als die der Platinsalze. Man bat also geringere Mengen von Flüssigkeit zu bearbeiten und die Trennung gelingt leichter.

Wenn bei Darstellung der jetzt so häufig gebrauchten Lithionsalze der vorher nach Schrötter geschmolzene Lepidolith statt mit $\mathrm{HCl}$ mit $\mathrm{SO}^{3}$ aufgeschlossen wird, so können die Fabriken den Chemikern schon reine Rubidium - und Cäsiumsalze liefern, vorausgesetzt, dass sie das Spectroskop dabei anwenden.

Zur Reinigung des käuflichen Kalium- und Cäsiumhaltigen Chlorrubidiums verwandelt man es mit Hülfe von Ammoniakalaun in die entsprechenden Alaune und trennt diese durch Umkrystallisiren. Man rechnet für die anzuwendende Menge Salz die äquivalente Menge von Ammoniakalaun, löst letzteren in so viel Wasser als er bei $170 \mathrm{C}$. zur Lösung braucht, setzt dann das Rubidiumund Cäsiumsalz zu, kocht auf bis zur Lösung des letzteren und lësst durch Erkalten krystallisiren. Die Krystallisation wird gestört, um kleine Krystalle zu erhalten. Diese werden abfiltrirt, mit kaltem' Wasser gewaschen und noch einmal umkrystallisirt. So gelingt es oft mit dieser zweiten Krystallisation schon ein reines Salz zu erhalten.

Es erklärt sich dies aus der Löslichkeit der Salze.

Es lösen sich nämlich bei $17^{\circ} \mathrm{C}$. in $10 \mathrm{Th}$. Wasser 0,619 Th. Cäsiumalaun $\mathrm{CsO}, \mathrm{SO}^{3}+\mathrm{Al}^{2} \mathrm{O}^{3}, 3 \mathrm{SO}+24 \mathrm{HO}$, 2,27 Rubidiumalaun $\mathrm{RbO}, \mathrm{SO}^{3}+\mathrm{Al}^{2} \mathrm{O}^{3}, 3 \mathrm{SO}^{3}+$ $24 \mathrm{HO}$,

12,7 Ammoniakalaun $\mathrm{H}^{4} \mathrm{NO}, \mathrm{SO}^{3}+\mathrm{Al}^{2} \mathrm{O}^{3}, 3 \mathrm{SO}^{3}$ $24 \mathrm{HO}$, (darin 1,74 Th. $\mathrm{H}^{4} \mathrm{NO}, \mathrm{SO}^{3}$ )

13,5 nalialaun $\mathrm{KO}, \mathrm{SO}^{3}+\mathrm{Al}^{2} \mathrm{O}^{3}, 3 \mathrm{SO}^{3}+24 \mathrm{HO}$, (darin 2,85 $\mathrm{Th} . \mathrm{KO}, \mathrm{SO}^{3}$ )

11,3 , schwefelsaures Kali $\mathrm{KO}, \mathrm{SO}^{3}$,

33,3 " Chlorkalium KCl, 
37,7 Th. Chlorammonium $\mathrm{H}^{4} \mathrm{NCl}$ und

50,0 , schwefelsaures Ammoniak $\mathrm{H}^{4} \mathrm{NO}, \mathrm{SO}^{3}$. (Wien. Sitzungsber. d. k. Akad.d. Wissensch. Math.-naturw. Classe. LI. Bd. III. H. Jahrg. 1865. Mdrz. II. Abth. S. 247 - 251.)

H. Ludwig.

\section{Zur Bestimmung des Kalkes als Aetzkalk; von Franz Stolba in Prag.}

Der Verfasser bedient sich zu seinen Versuchen einer aus sechs gewöhnlichen einfachen Bunsen'schen Brennern construirten Gaslampe, in welcher die Brenner in einem Kreise von 112 Millim. Durchmesser stehen. Um die Hitze gehörig zusammen zu halten, wendet derselbe einen bereits vielfach empfohlenen Schornstein von Eisen oder Thon zum Zusammenhalten der Flammen an und setzt auf denselben den Platintiegel im Platintriangel. Quantitäten von Kalk, welche $1 \mathrm{Grm}$. nicht übersteigen, erhitzt man 10-15 Minuten lang im vollen Gasstrome und kehrt dann mittelst eines Platindrähtchens, welches am Ende gebogen ist, den lose zusammenhängenden Kuchen der Art um, dass die Theile, welche früher unten lagen, nun oben auf kommen, glüht abermals 10-15 Minuten, lässt erkalten und wiegt. Auf diese Weise erhielt der Verf. bei der erwähnten Menge den Kalk stets vollkommen ätzend. Es zeigte sich bei wiederholtem Glühen das Gewicht als constant und Salzsäure entwickelte keine Spur von Kohlensäure.

Mengen über 1-2 Grm. kohlensauren Kalkes werden auf angegebene Art nicht vollständig ätzend, weil die Schicht zu hoch ist. In diesem Falle hilft man sich, indem man den kohlensauren Kalk gleichförmig a m Boden und an den Seiten des Platintiegels vertheilt, so dass es sich bis auf einige Linien dem Rande des Tiegels nähert, was am besten mittelst eines runden glatten Glasstäbchens geschieht. Etwa am Glasstäbchen befindliche Theile des kohlensauren Kalkes werden mit einem Platindrahte entfernt oder an einem 1/4 Quadratzoll grossen Streifen von schwedischem Filtrirpapier abgewischt, worauf man das Papier am Deckel verbrennt. Mengen über 2 Grm. ätzend zu brennen, bält der Verf. das Gasgebläse für nötbig.

Die sich dabei aufdringende Frage, ob bei dieser Bestimmung die Anwendung des Gases nicht Fehlerquellon verursacht, hält der Verf. für wohl zu beachten und 